chosen zones must not be less than 10 . The resolution values that had been calculated by the chromatograms obtained in different laboratories satisfied the method requirements. The suggested method can be used for identification of Aerva lanata herb in routine analyses. This method has been included into the draft version of "Krasnogorskleksredstva" House monograph on Aerva lanata herb.

\title{
NEUROPROTECTIVE EFFECTS OF CORTEXIN, A DRUG DERIVED FROM THE BRAIN CORTEX
}

\section{() Shabanov Petr D.}

Military Medical Academy, 6, Acad. Lebedev street, St.Petersburg, 194044, Russia Institute of Experimental Medicine NWB RAMS, 12, Acad. Pavlov street, St.Petersburg, 197376, Russia; e-mail: pdshabanov@mail.ru

According to the conception of neuroprotection widely spreaded in neurology, neuroprotectors are shown to propose a number of specific requirements (Shabanov P. D., 2009): 1) to have nootropic or nootropic-like effects consisting of an ability to improve the resistance of the organism, and the central nervous system in particular, to action of different damaged factors simultaneously with maintenance or enhancement of the higher functions of the brain; 2) to optimize metabolic activity of neural cells in action of aggressive factors of the environment; 3 ) to recover of impaired metabolism and energy deficit in neural cells; 4) to have anti-oxidative (anti-free radicals) effect; 5) to activate directly neurotrophic processes by means of stimulation of sprouting and formation of neurotrophic factors; 6 ) to prevent neuronal degeneration due to different pathological processes (autoimmune, toxic, aged, accelerated apoptosis). Among the candidates reviewed for these positions it should be consider cortexin, a peptide organ/tissue drug derived from the porcine brain cortex. The comparative investigation of a number peptide drugs (brain tissue drugs cerebrolysine and cortexin, synthetic peptides semax, selank, deltaran, noopept, dilept) in experimental and clinic conditions showed their high neuroprotective activity. It was registered both after administration of drugs into the brain ventriculi and after systemic (intraperitoneal) administration. The direction of effects after both types of administration was similar that indicated the peptide drugs penetrated the brain blood barrier. Moreover, the peptides were effective in diminution of subsequences following prolong social isolation, activation of the stress/antistress systems in early ontogeny (Shabanov P. D. et al., 2009), in experimental ischemia of the brain after occlusion of both carotid arteries, in traumatic toxicosis, after mechanic brain injury (Zarubina I. V. et al., 2011), in elevated seizure activity (Gulyaeva N.V. et al., 2011). In clinic, cortexin, cerebrolysine, semax and noopept were effective in postabstinent period in patients with alcoholism, in heavy somatic patients suffered from asthenic syndrome, in brain damaged patients, in children with seizure syndrome, after intoxication with neurotropic poisons, for acceleration of acclimatization in quick changes of meteorological conditions of environment (Ganapolskii V. P., 2011). It is important to emphasize that brain tissue drugs, cortexin in particular, were clinically more effective than synthetic neuropeptides, though in the experimental conditions their pharmacological activity often was higher than of brain tissue drugs. Besides, all drugs of metabolic type of action possessed an individual sensitivity to treatment when a half of patients reacted on drug brightly, but another one reacted by minimal improvement. Therefore, peptide neuroprotectors should be consider as perspective drugs for the treatment of many diseases of the central nervous system where both neuronal degeneration and reduction of metabolic status of the neural cells are the leading pathophysiological factors. 